\title{
Magnetically induced micropillar arrays for an ultrasensitive flexible sensor with a wireless recharging system
}

\author{
Libo Gao ${ }^{1,2,3 \dagger^{*}}$, Ying $\mathrm{Han}^{4 \dagger}$, James Utama Surjadi ${ }^{4}, \mathrm{Ke} \mathrm{Cao}^{1,2}$, Wenzhao Zhou ${ }^{3}$, Hongcheng Xu ${ }^{1,2}$, \\ Xinkang $\mathrm{Hu}^{1,2}$, Mingzhi Wang ${ }_{5,6^{*}}^{1,2}$, Kangqi Fan ${ }^{1,2}$, Yuejiao Wang ${ }^{3,4}$, Weidong Wang ${ }^{1,2,5^{*}}$ and \\ Horacio D. Espinosa ${ }^{5,6^{*}}$
}

\begin{abstract}
Significant efforts have been devoted to enhancing the sensitivity and working range of flexible pressure sensors to improve the precise measurement of subtle variations in pressure over a wide detection spectrum. However, achieving sensitivities exceeding $1000 \mathrm{kPa}^{-1}$ while maintaining a pressure working range over $100 \mathrm{kPa}$ is still challenging because of the limited intrinsic properties of soft matrix materials. Here, we report a magnetic field-induced porous elastomer with micropillar arrays (MPAs) as sensing materials and a well-patterned nickel fabric as an electrode. The developed sensor exhibits an ultrahigh sensitivity of $10,268 \mathrm{kPa}^{-1}$ $(0.6-170 \mathrm{kPa})$ with a minimum detection pressure of $0.25 \mathrm{~Pa}$ and a fast response time of $3 \mathrm{~ms}$ because of the unique structure of the MPAs and the textured morphology of the electrode. The porous elastomer provides an extended working range of up to $500 \mathrm{kPa}$ with long-time durability. The sophisticated sensor system coupled with an integrated wireless recharging system comprising a flexible supercapacitor and inductive coils for transmission achieves excellent performance. Thus, a diverse range of practical applications requiring a low-to-high pressure range sensing can be developed. Our strategy, which combines a microstructured high-performance sensor device with a wireless recharging system, provides a basis for creating next-generation flexible electronics.
\end{abstract}

Keywords: high sensitivity, pressure sensor, supercapacitor, wireless recharging, flexible electronics

\section{INTRODUCTION}

A highly sensitive flexible sensor is a basic unit used to construct wireless sensor networks (WSNs) for building Internet-of-Things (IoT) devices in health monitoring, robotic control, and electronic skin [1-10]. However, significant challenges are posed by the inherent trade-off between sensitivity and working range and other related figures of merit, e.g., response time, mechanical flexibility, and durability, because of the intrinsic "soft" mechanical property of the matrix materials typically used in flexible sensors [11,12]. Therefore, sensing structures/materials should be enhanced to explore other relevant aspects influencing a sensor's performance.

Flexible pressure sensors have been widely investigated. Based on their sensing mechanisms, they can be typically classified into four types: piezoresistive, piezocapacitive, piezoelectric and triboelectric [13]. In the case of piezoresistive pressure sensors, previous studies primarily focused on improving their sensitivity via two strategies [14-16]: (1) modification of the contact area between facing conductive components (i.e., sensing materials and electrodes) by building unique structures (e.g., micropillars, microdomes or micropyramids) either on sensing material surfaces or on electrode patterns; (2) rationally designing geometric topological structures to enhance the intrinsic compliance of sensing materials to induce localized geometrical deformation when they are subjected to

\footnotetext{
${ }^{1}$ School of Mechano-Electronic Engineering, Xidian University, Xi'an 710071, China

${ }^{2}$ CityU-Xidian Joint Laboratory of Micro/Nano-Manufacturing, Shenzhen 518057, China

${ }^{3}$ Nano-Manufacturing Laboratory (NML), Shenzhen Research Institute of City University of Hong Kong, Shenzhen 518057, China

${ }^{4}$ Department of Mechanical Engineering, City University of Hong Kong, Kowloon 999077, Hong Kong SAR, China

${ }^{5}$ Department of Mechanical Engineering, Northwestern University, Evanston, Illinois 60208, USA

${ }^{6}$ Theoretical and Applied Mechanics Program, Northwestern University, Evanston, Illinois 60208, USA

These authors contributed equally to this paper.

*Corresponding authors (emails: lbgao@xidian.edu.cn (Gao L); wangwd@mail.xidian.edu.cn (Wang W); espinosa@northwestern.edu (Espinosa HD))
} 
subtle pressure changes. Despite recent progress on previously developed methods, achieving ultrahigh sensitivities of over $1000 \mathrm{kPa}^{-1}$ while simultaneously keeping a working range of over $100 \mathrm{kPa}$ (high pressure) remains challenging.

A power source is another material essential for flexible sensors. Power sources should exhibit high-energy storage density while accommodating mechanical deformation with minimal loss in conformability and energy loss $[1,17]$. Supercapacitors present an attractive solution because of their recharging capability with a lower energy loss than conventional batteries, as demonstrated in various flexible sensors, including the one described in our previous work [18-22]. However, previous studies disregarded the fact that an effective wireless charging system without a direct physical connection is necessary to avoid a modulus mismatch between an energy storage circuitry and a sensing unit and thus achieve the portability of integrated devices [17].

In this study, two key strategies were combined to develop a revolutionary high-performance wireless sensor with an ultrahigh sensitivity of over $1000 \mathrm{kPa}^{-1}$ and a broad working range $(>100 \mathrm{kPa})$. A porous architecture was introduced to bulk elastomers to enhance compres- sibility. Micropillar arrays (MPAs) were then grown on a foam via magnetic field induction to decrease the contact areas with the sensor electrode. The resulting device obtained a sensitivity of $10,268 \mathrm{kPa}^{-1}$ at $0.6-170 \mathrm{kPa}$ and a working range of up to $500 \mathrm{kPa}$. The device was further assembled with a wireless recharging system, which was composed of a flexible symmetrical supercapacitor with an energy density of $0.011 \mathrm{~mW} \mathrm{~h} \mathrm{~cm}^{-2}$ and rationally designed fast-charging wireless inductive coils for transmission. With this design, a wired external power was no longer necessary. The developed sensor system was the smart configuration of wireless-charging energy-storage sensors with diverse practical applications ranging from low pressures $(0-10 \mathrm{kPa})$ to moderate $(10-100 \mathrm{kPa})$ and high pressures $(>100 \mathrm{kPa})$.

\section{RESULTS AND DISCUSSION}

\section{Device design and structural characterization}

A wearable sensor with high sensitivity needs to capture physiological signals, such as pulse rate, walking, and running speed, by building a sensor network to address the demands of health monitoring systems (Fig. 1a). In this regard and in consideration of the diverse range of

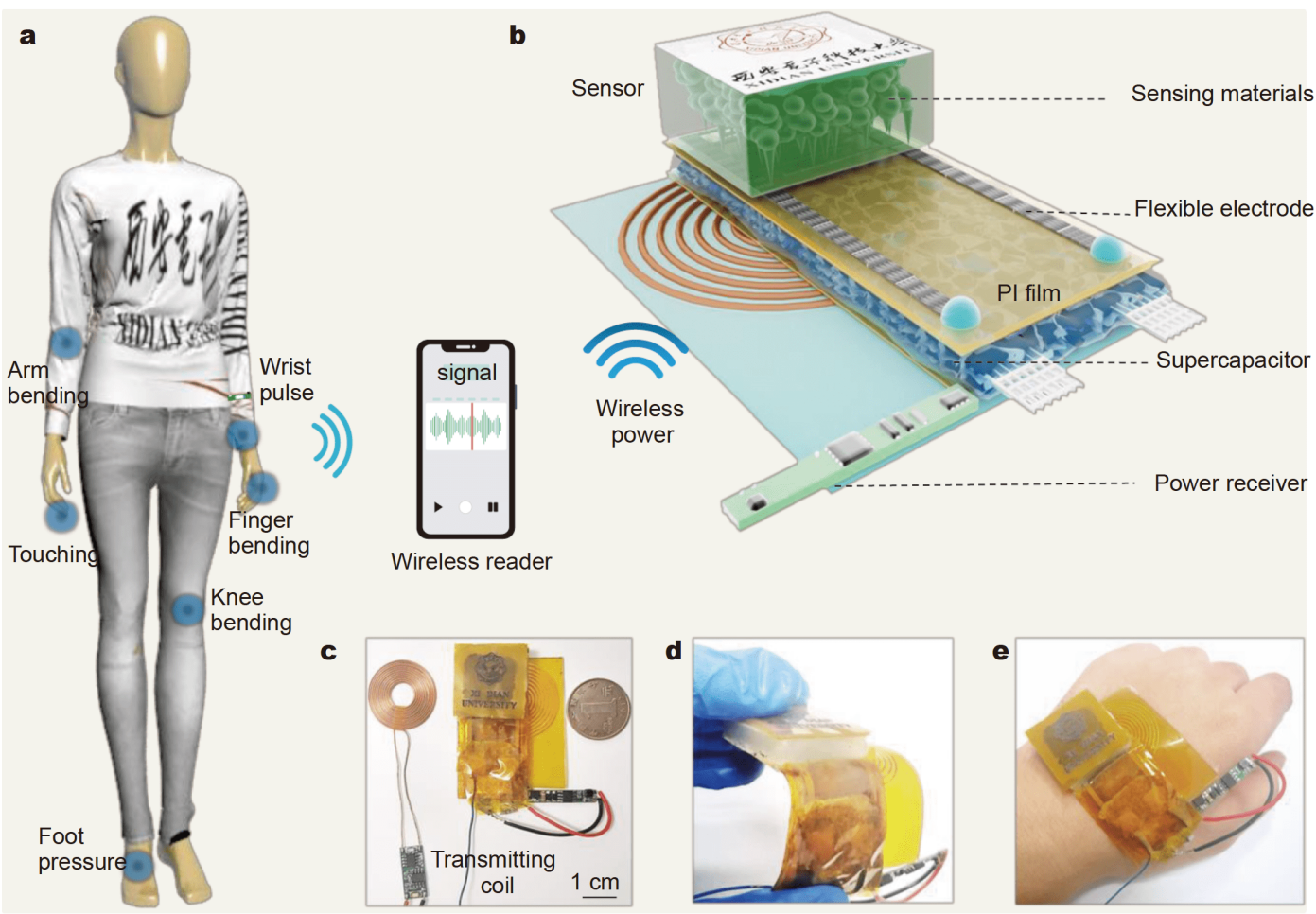

Figure 1 Flexible sensor layout and design. (a) Schematic of the pressure sensor's applications. (b) Exploded view layout of various layers of the sensor. (c) Optical image of the integrated sensor. (d) Bending state of the device. (e) Conformal wearability of the sensor. 
application requirements, a sensor that can detect an extensive pressure range from low pressures $(0-10 \mathrm{kPa})$ to moderate $(10-100 \mathrm{kPa})$ and high pressures $(>100 \mathrm{kPa})$ is highly desirable. Furthermore, a wireless reader and recharging system should have user mobility and convenience. In this study, an ultrasensitive flexible sensor integrated with a wireless recharging system (Fig. 1b) is rationally designed with all the above requirements. The sensor consists of three parts: a sensing material with MPAs and micropores, a flexible electrode, and an ultrasoft encapsulating silicone elastomer. The wireless charging system is mainly composed of two parts: a wireless charging power receiver coupled with an electromagnetic flux from a primary coil (transmitting coil) and a quasi-solid supercapacitor. Inside a polydimethylsiloxane (PDMS) elastomer is a receiving copper coil that allows bending to improve the comfort for wearers (Fig. S1). Furthermore, a supercapacitor with high-energy density is employed by directly growing $3 \mathrm{D}$ carbon electrode materials with a hierarchical porous structure on a stainless steel wire. The image of the entire device with a size of $3 \mathrm{~cm} \times 5 \mathrm{~cm}$ is shown in Fig. 1c, and the flexibility and wearable ability of the assembled device are presented in Fig. 1d, e. The size of the whole device can be easily controlled, as discussed below.

The proposed sensor working mechanism is illustrated in Fig. 2a. In our design, sensitivity is defined as $S=$ $\left(\Delta I / I_{0}\right) / \Delta P$, where $\Delta I$ is the relative current change, $I_{0}$ is the initial current without any applied pressure, and $\Delta P$ is the corresponding pressure variation. $I_{0}$ should be as low as possible, and $\Delta I$ should be enhanced to increase sensitivity. Therefore, in the design concept, the sharp edge of MPAs induces a higher initial resistance because of the low contact area. Once pressure is applied, MPAs are easily bent, thereby rapidly increasing conductivity. The continuous application of pressure also results in an increasing number of contact points between the adjacent cells inside, further enhancing conductivity because of the
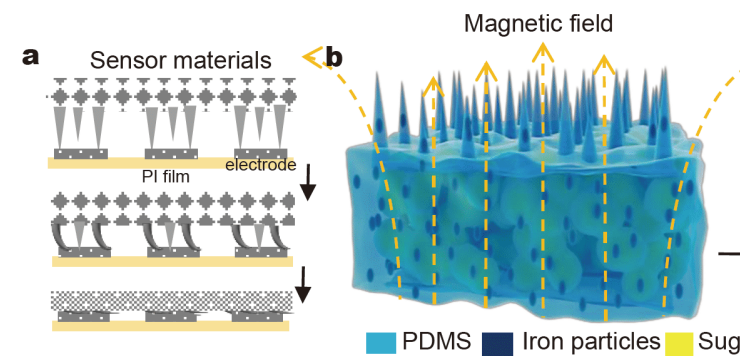

c

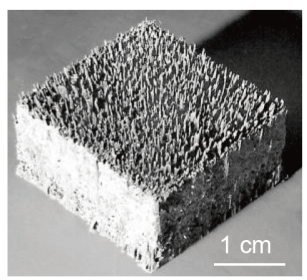

g

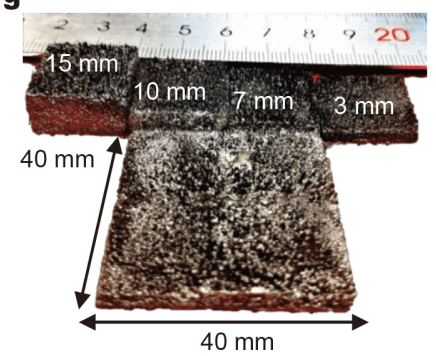

d

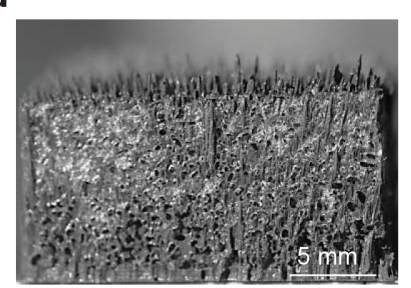

h

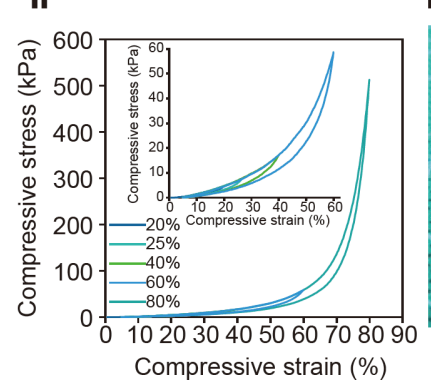

i

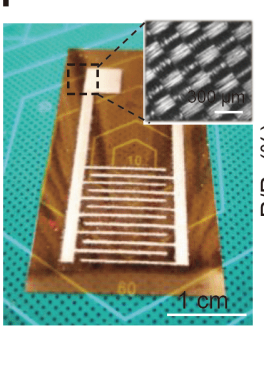

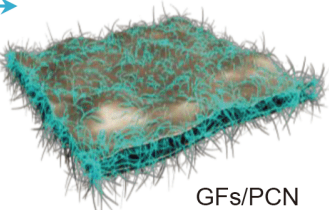

f
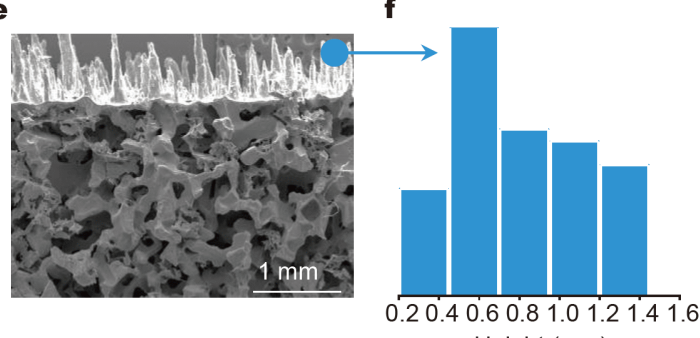

j Height $(\mathrm{mm})$

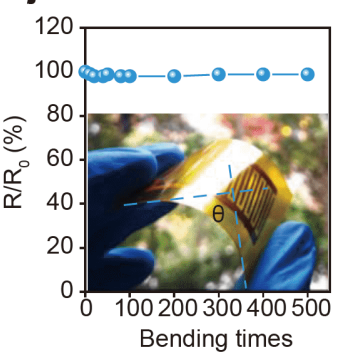

Figure 2 Structural characterization of the sensing materials and electrode. (a) Illustration of the sensor mechanism. (b) Processing of the porous elastomer with micropillars. (c) 3D view of the porous elastomer and the corresponding enlarged view of the pores and pillars. (d) Side view of the sample and (e) its corresponding SEM image. (f) Height distribution of the micropillars. (g) Large-scale fabrication of the sample with various sizes. (h) Loading-unloading experiments with various compressive strains. (i) Digital optical image of the flexible electrode. (j) Bending deformation of the flexible electrode and its corresponding resistance change. 
increased contact area. Lastly, the synergistic effect of MPAs and foams plays a major role in improving conductivity.

The two main structures (i.e., MPAs and foams) are carefully integrated to achieve this goal. Sugar as a sacrificial template and a magnetic field was used to grow Fe-loaded MPAs directly on the surface of foams (Fig. 2b). The three-dimensional (3D), side, and top views of the obtained sample are shown in Fig. 2c, d and Fig. S2, respectively. As clearly observed in these digital optical images, uniform and ordered cone-shaped MPAs are directly grown on the porous matrix by inducing the $\mathrm{Fe}$ microparticles under a magnetic field (Fig. S3). This result is further revealed by microstructural characterization (Fig. 2e). The MPAs exhibit a broad height distribution ranging from 0.2 to $1.4 \mathrm{~mm}$ (Fig. 2f), which can be exploited to enhance the sensor's sensitivity and working range. Additionally, the open-cell PDMS matrix has a hierarchically porous architecture with a size ranging from several micrometers to millimeters. The unique structures of the hierarchical MPAs and the porous PDMS matrix significantly enhance the sensor's performance. For example, Song et al. [23,24] used the bulk porous PDMS to develop an all-in-one self-powered sensor with a high performance. In another study, the obtained flexible architecture was coated with a hybrid film composed of graphite flakes and a pulp cellulose nanofiber (GF/PCN) film to achieve the desired conductivity. The PCN film was added to a solution containing GFs to prepare a more stable colloidal dispersion than a pure solution containing GFs (Fig. S4). The PCN anchored on GFs improves not only the dispersion but also the mechanical strength of the composite via the formation of hydrogen bonds between GFs and PCN [25].

Transmission electron microscopy (TEM) imaging (Fig. S4) further revealed the homogeneous dispersion of the PCN on the GFs. Therefore, the GF/PCN could be uniformly coated on the PDMS matrix (Fig. S5). The Fe content is necessary to grow MPAs with uniformity and control the height of micropillars (Figs S6 and S7). An insufficient $\mathrm{Fe}$ content (0.2 and 0.4) could lead to nonuniform sparsely grown MPAs that resulted in an unstable performance of the sensor. Excessive Fe content (0.8) led to highly dense and compacted MPAs with a large diameter, thereby decreasing the initial contact resistance and consequently reducing the sensor sensitivity. Moreover, the viscosity of the composite matrix would increase as the concentration of the $\mathrm{Fe}$ microparticles increased; this phenomenon could cause an incomplete penetration of the template (Fig. S8). In this regard, a weight ratio of 0.6 was identified to be optimal and employed in this study. This method could be easily extended to large-scale applications, and the thickness of the film could be easily tuned to make the whole device more flexible and portable. This finding was illustrated by various dimensional structures manufactured with thickness dimensions ranging from 3 to $15 \mathrm{~mm}$ and architectures with dimensions of $40 \mathrm{~mm} \times 40 \mathrm{~mm}$ (Fig. $2 \mathrm{~g}$ ).

The as-prepared sample was mechanically robust, demonstrating excellent durability when it was subjected to tensile, bending, squeezing, and twisting tests (Fig. S9). This observation was verified through the quantitative analysis of the loading-unloading cycling compression test (Fig. 2h). Successive loading curves followed the trend of the initial loading curve, thereby maintaining the maximum stress point over the entire range. This result indicates a perfect strain memory effect and an excellent recoverability. Interestingly, sensing materials showed strong magnetic attraction even at a considerable distance from a magnet (Fig. S10).

In addition to sensing materials, a flexible electrode is an important part of the system that plays an equally significant role in enhancing the sensitivity and working pressure range of the sensor, which was already demonstrated in our previous study [26]. In our investigation, the flexible electrode (nickel fabrics) was manufactured in accordance with a novel "subtracting and transferring" protocol, with designed patterns on a polyimide (PI) or polyethylene terephthalate PET film (thickness = $100 \mu \mathrm{m}$ ), which served as the working electrode similar to our previous sensor embodiment [26] (Fig. 2i and Figs S11, S12). The detailed manufacturing method can be found in Supplementary information. We found that the uniformly textured structure could help further enhance the sensitivity of the sensor. Moreover, the electrode exhibits a high flexibility and a superior electromechanical stability ( $>98 \%$ retention) even after $90^{\circ}$ bending for 500 times (Fig. 2j). Overall, these findings imply that flexible porous sensing materials with MPAs and well-patterned and integrated electrode materials exhibit a reliable electrochemical performance and therefore show significant potential for application in flexible electronics.

\section{Sensor characterization}

The sensor performance was characterized in terms of sensitivity, working range, and response time (Fig. 3 and Figs S13, S14. The sensor displays three well-defined regions with sensitivities of $2010.5 \mathrm{kPa}^{-1}(0.00025-0.6 \mathrm{kPa})$, 

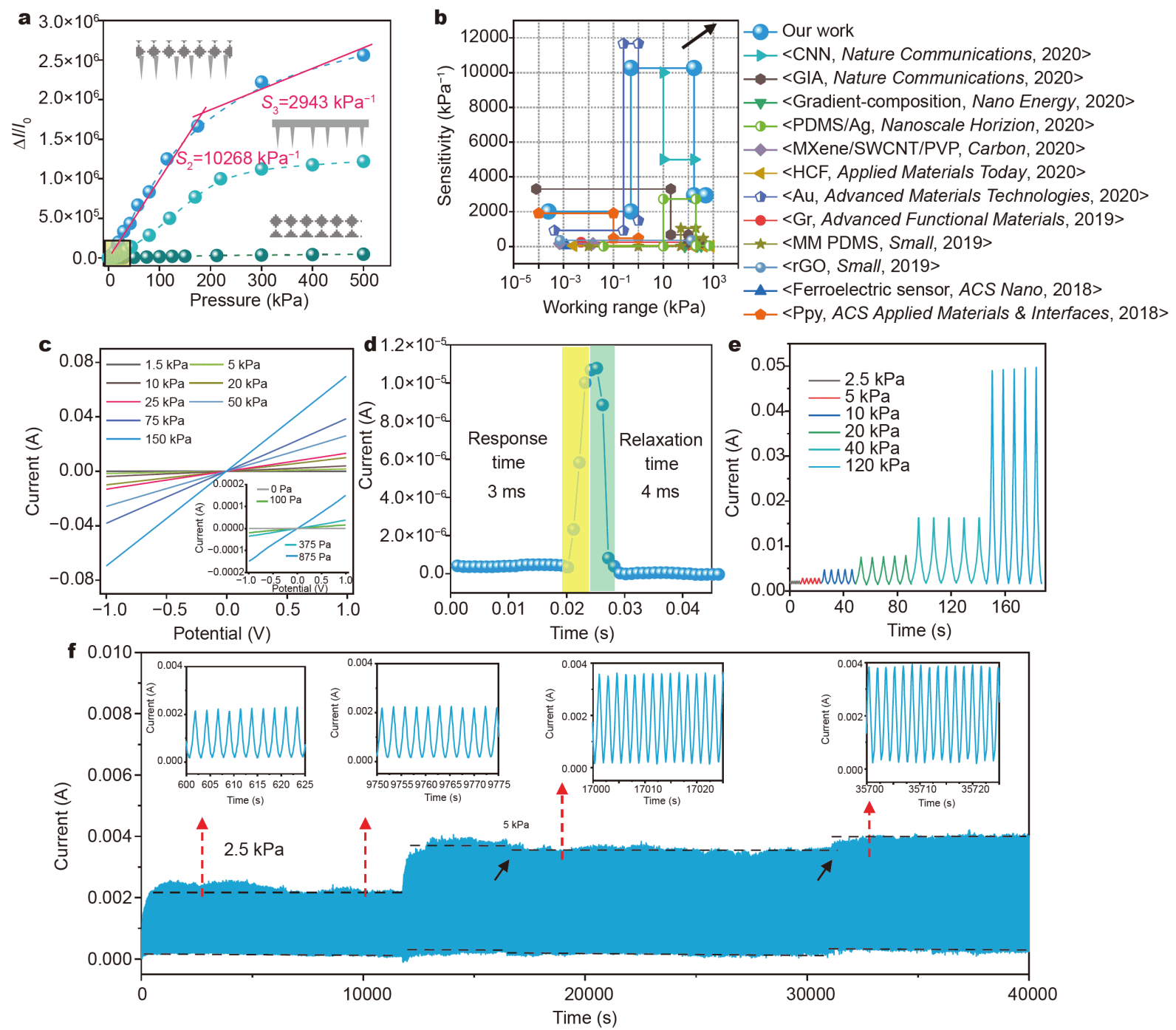

Figure 3 Characterization of the sensor. (a) Normalized current variation versus pressure change. (b) Comparison of the created sensor with other flexible sensors. (c) $I-V$ plots of the sensor under various applied pressures. (d) Sensor response and relaxation time curves. (e) Current signatures under increasing pressures. (f) Long-time sensor response under 2.5 and $5 \mathrm{kPa}$.

$10,268 \mathrm{kPa}^{-1}(0.6-170 \mathrm{kPa})$, and $2943 \mathrm{kPa}^{-1}(170-500 \mathrm{kPa})$ and a remarkably broader linear working range than that of bare foam or micropillars (Fig. 3a). This high sensitivity is primarily derived from the low contact area (high contact resistance) induced by the MPAs. This ultrahigh initial resistance could improve sensitivity and reduce power consumption in a standby mode. This balanced combination of the ultrahigh sensitivity and wide linear working range outperforms previously developed sensors [11,27-37], as illustrated in Fig. 3b. This finding was further confirmed by the stable performance exhibited by the sensor at various applied voltages $(V)$ (Fig. 3c).

Response time is crucial because it determines the sensor's ability to provide rapid feedback to external stimuli. The sensor not only showed a fast response time of $3 \mathrm{~ms}$ but also exhibited a relaxation time of $4 \mathrm{~ms}$, demonstrating its great potential for practical application. A dynamic test was further conducted on the sensor under various applied pressures ranging from 2.5 to $120 \mathrm{kPa}$. The linear response (Fig. 3a) between $\Delta I / I_{0}$ and $\Delta P$ implied stable sensitivity, which was further verified in current-time $(I-t)$ measurements at various pressures (Fig. 3e). Long-term durability is another essential feature relevant to the practical applications of the sensor. Therefore, the sensor's cycling performance was assessed under various pressures of 2.5 and $5 \mathrm{kPa}$ for $12,000 \mathrm{~s}$ 
(5000 cycles) and $28,000 \mathrm{~s}$ (15,000 cycles), respectively (Fig. 3f). These results show a negligible decay in the sensor's performance during the whole testing process. In Fig. S15, the conductivity increased rapidly when a magnetic field ranging from 0 to $100 \mathrm{mT}$ was applied because of the magnetic property of the sensor. Then, the conductivity gradually changed from 100 to $400 \mathrm{mT}$ because the magnetic field would initially cause the MPAs to bend, inducing a rapid contact change. Conversely, the deformation of the PDMS foam was mainly responsible for the change in conductivity in the subsequent part (i.e., $100-400 \mathrm{mT}$ ).

\section{Sensor applications}

The performance of the sensor was carefully investigated when it was employed in the detection of pulse rate, finger tapping, foot pressure, and human-machine interaction (HMI) to demonstrate the sensor's applicability to a wide range of pressures (i.e., from low to high pressure). When the sensor was placed on the wrist of a subject, it successfully monitored the percussion wave (P), tidal wave (T), and dicrotic wave (D) while revealing a pulse rate of $68 \mathrm{~min}^{-1}$ (Fig. 4a). In another demonstration, tapping pressure was employed to continuously adjust the intensity of a lamplight (Fig. $4 \mathrm{~b}$ and Movie S1). Conversely, almost no energy was consumed in the standby mode.

Wireless communication is essential for IoT applications. Therefore, the sensor with a Bluetooth device that could successfully transmit the force information was configured directly into a smartphone (Fig. $4 \mathrm{c}$ and Movie S2). With the long-distance transmission feature of the Bluetooth device, the sensor could serve as an optimal terminal unit device in WSNs. Another application of interest is foot pressure mapping that has been widely used for medical diagnostics and sports training [38]. The developed sensor was successfully used to quantify foot pressure and monitor a standing subject. Therefore, the sensor could be used for foot pressure mapping (Fig. 4d). Additionally, the sensor arrays could be easily fabricated (Fig. S16).

HMI is an important part of the modern IoT. Hence, the sensor's applicability to HMI scenarios was explored. The performance of the flexible sensors under finger bending was quantitatively tested (Fig. 4e). During the bending of the finger from $0^{\circ}$ to $90^{\circ}$, the sensor displayed ultrahigh sensitivity and flexibility because of the enhanced contact area. Our sensor was assembled with a robot hand through a microcontroller (Fig. 4f), and the robot hand was successfully manipulated by synchroniz- ing its movement with the subject's movement via the developed sensor. In conclusion, these demonstrations confirm that the created flexible sensor could address a number of applications ranging from low pressures to high pressures.

\section{Flexible supercapacitor device}

The energy storage module of a device determines the long-term durability of a sensor and its downtime, i.e., how fast it can be charged to drive the sensor. In our study, a hierarchically porous graphene/carbon nanotube (CNT) hybrid structure was built onto a stainless steel mesh to construct electrode materials. In Fig. $5 a-d$, the electrode has a uniform interconnected set of open macro and micropores with sizes ranging from 10 to $800 \mu \mathrm{m}$ across the whole structure. This characteristic facilitates electrolyte penetration into the inner part to fully exploit the potential of the graphene electrode. Given the high conductivity of the homogeneously distributed CNTs anchored by the graphene substrate (Fig. 5d) and fully reduced graphene [22] (Fig. S17), the energy storage capacity and rate capability were greatly enhanced. As a demonstration, the assembled quasi-solid symmetrical device (Fig. S18) exhibited a typical electric double layer capacitor behavior at various scan rates and current densities (Fig. 5e, f). The 3D porous structure was nearly vertical to the real axis in the low-frequency portion of the Nyquist plot, demonstrating the fast transport of ions into the electrode (Fig. $5 \mathrm{~g}$ ). The areal capacitance of the assembled device held $75 \%$ (from 76.5 to $60.6 \mathrm{mF} \mathrm{cm}^{-2}$ ) retention when the current density increased by 20 -fold from 2 to $40 \mathrm{~mA} \mathrm{~cm}^{-2}$. This result suggests that the device could be fully charged even under a large charging current (Fig. 5h). Furthermore, the device's exceptional capacitive performance could readily drive a hygrothermograph (Fig. S18) and outperform other reported graphene-based micro-supercapacitors, such as functionalized holey graphene $\left(6.41 \mathrm{mF} \mathrm{cm}^{-2}\right)$ [39], vertical graphene $\left(9 \mathrm{mF} \mathrm{cm}^{-2}\right) \quad[40], \quad \mathrm{N}$-graphene $\left(63 \mu \mathrm{F} \mathrm{cm}^{-2}\right)$ [41], reduced grapheme oxide ( $\left.\mathrm{rGO}\right) /$ single wall CNTs (SWCNTs) $\left(30.76 \mathrm{mF} \mathrm{cm}^{-2}\right)$ [42], vertically aligned graphene $\left(7.3 \mathrm{mF} \mathrm{cm}^{-2}\right)$ [43], laser rGO $\left(0.5-2.5 \mathrm{mF} \mathrm{cm}^{-2}\right)$ [44], graphene paper $\left(22.3 \mathrm{mF} \mathrm{cm}^{-2}\right)$ [45], printed graphene $\left(0.7 \mathrm{mF} \mathrm{cm}^{-2}\right)$ [46], 3D cellular graphene $\left(\sim 6 \mathrm{mF} \mathrm{cm}^{-2}\right)$ [47], and MXene $\left(23.6 \mathrm{mF} \mathrm{cm}^{-2}\right)$ [48] (Fig. 5i). The maximum energy density of $0.011 \mathrm{~mW} \mathrm{~h} \mathrm{~cm}^{-2}$ with a corresponding power density of $0.00994 \mathrm{~mW} \mathrm{~cm}^{-2}$ was achieved, and these values were significantly superior to many previous findings (Fig.5j) [49-53]. The device could successfully power the elec- 

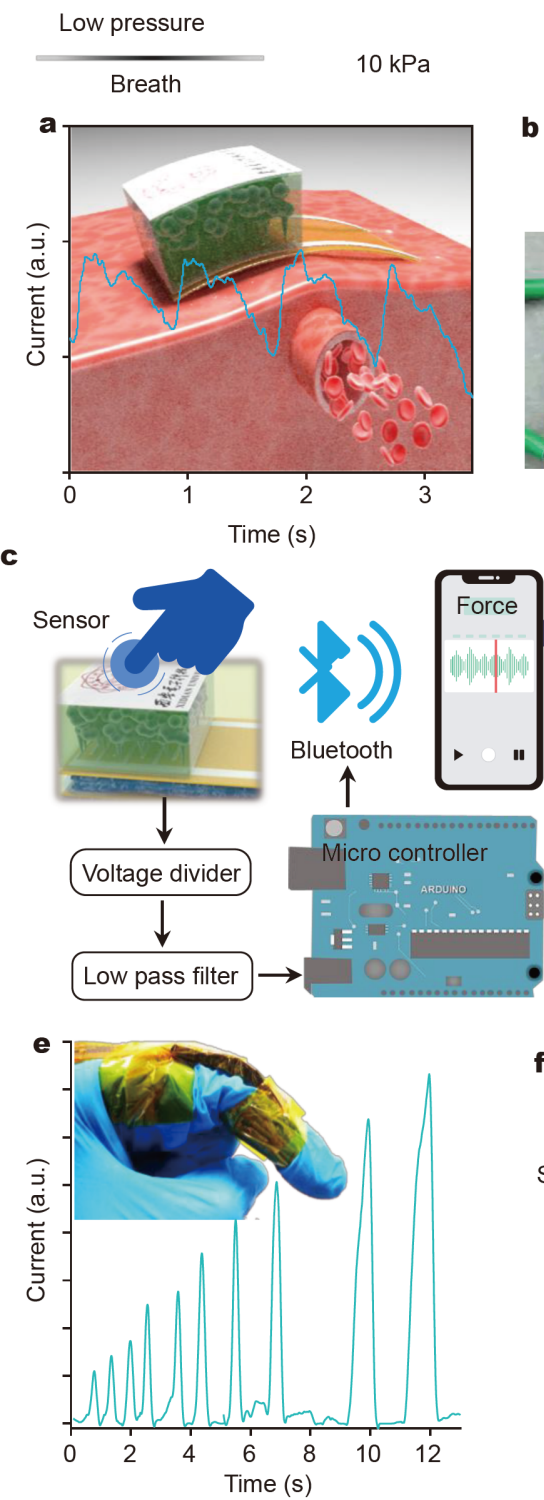

b
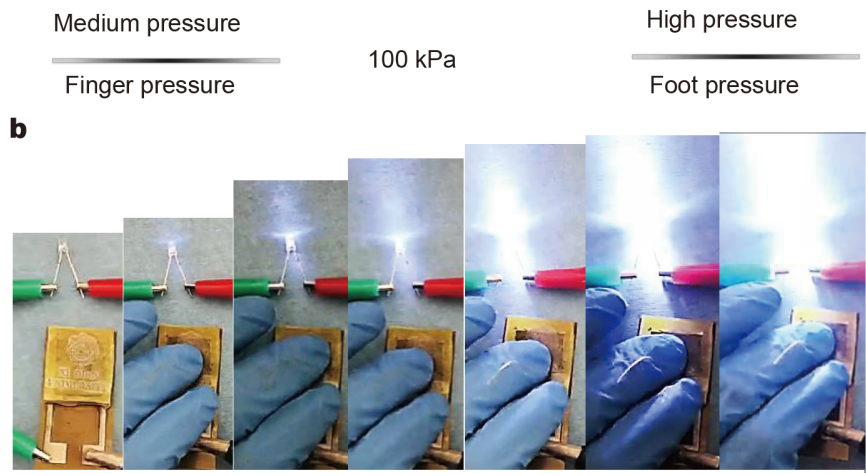

d
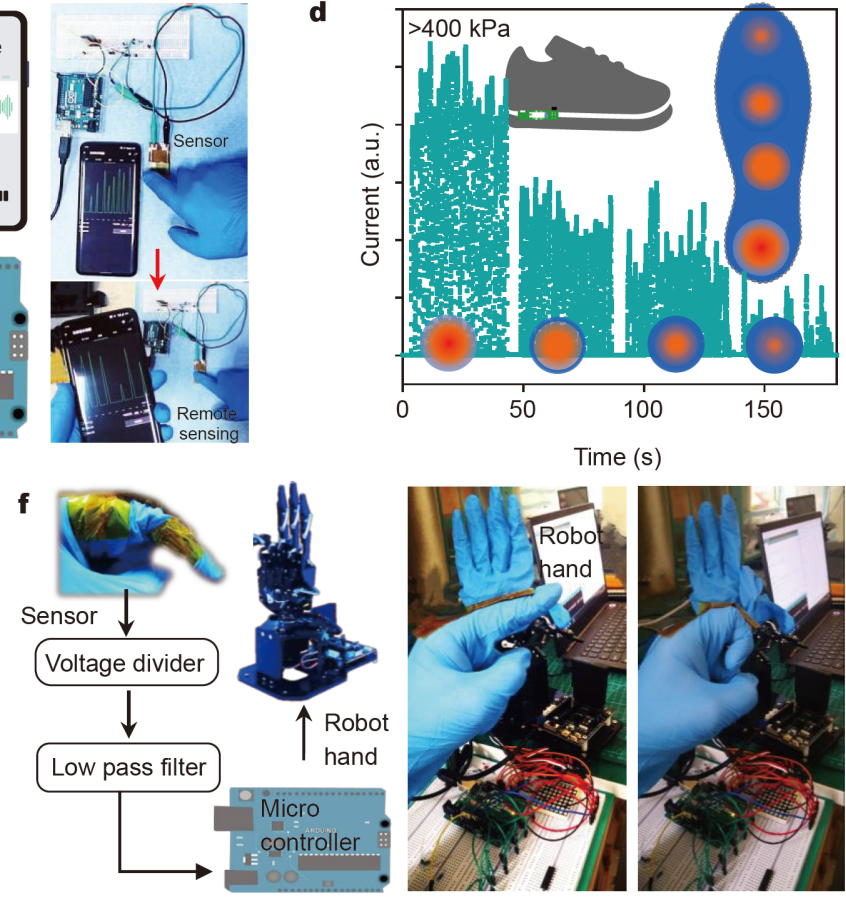

Figure 4 Practical applications of the sensor ranging from low pressure to high pressure. (a) Pulse rate monitoring. (b) Luminance control by finger tapping. (c) Wireless signal transmission to a cell phone. (d) Foot pressure mapping characterized by the pressure sensor. (e) Current variations under different bending degrees. (f) HMI application of the sensor.

tronics without having short circuits even when it was subjected to bending from $30^{\circ}$ to $180^{\circ}$. Therefore, it has excellent mechanical robustness and flexibility with negligible deterioration in performance (Fig. $5 \mathrm{k}$ and Fig. S17). The supercapacitor device retained $94.64 \%$ capacitance even after 3000 long-term cycling tests (Fig. S18). When two devices were connected in parallel (2P) or series (2S), performance reproducibility was confirmed (Fig. S19). The superior energy density of the flexible energy storage device serves as a solid foundation for driving the sensor, and the stable electromechanical performance guarantees its wearable capability.

\section{Flexible sensor integration with wireless recharging systems}

For practical applications, wireless power transmission is preferred, and the demand for this transmission is higher than that for wired connections because it not only provides convenience but also prevents damage to flexible sensors because of its flexible features [17]. In many practical cases, for instance, implanted pressure sensors driven by a supercapacitor equipped with a wireless 
a
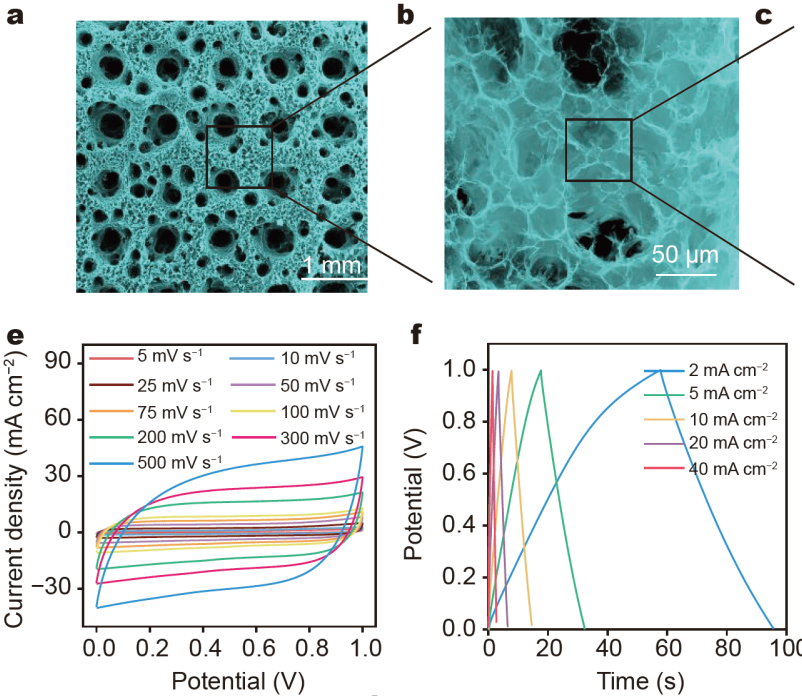

$\mathbf{f}$
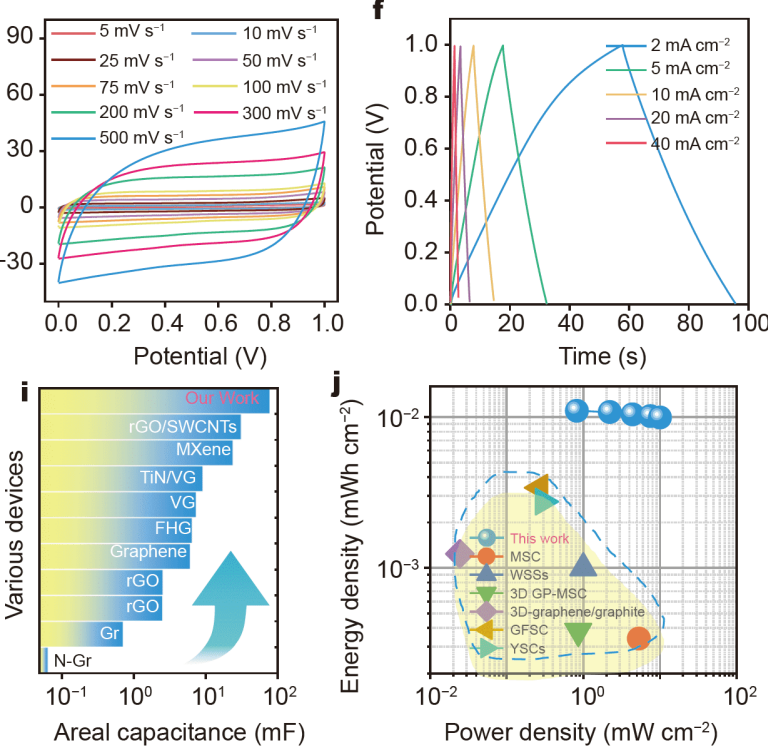
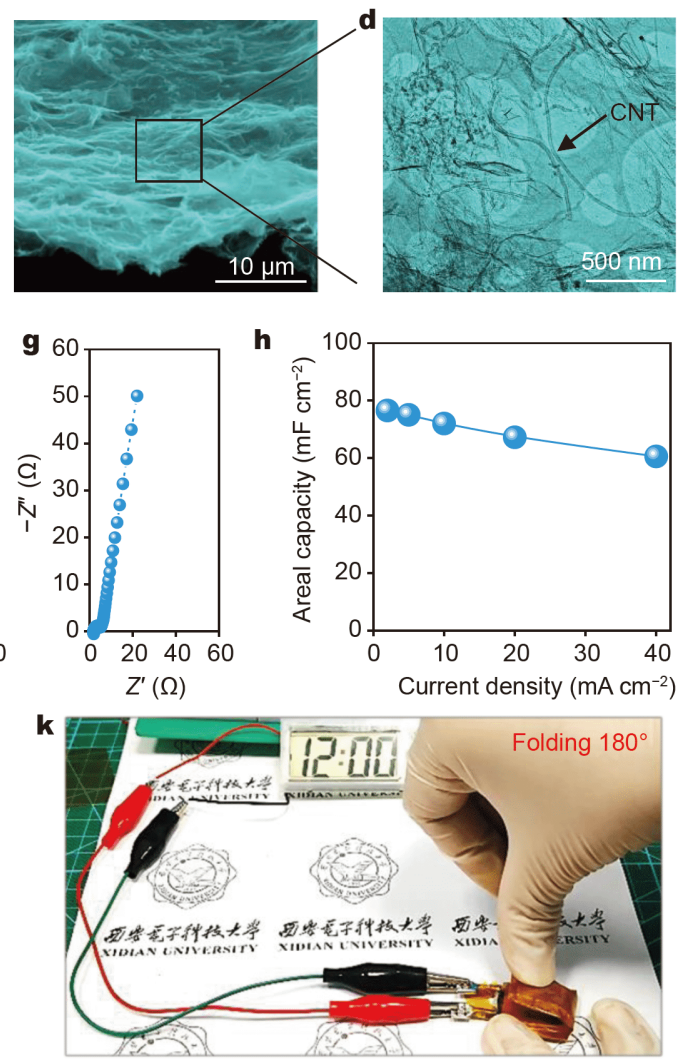

Figure 5 Characterization of the supercapacitor. (a-d) Image of the hybrid graphene/CNT supercapacitor hierarchically porous structure. (e) $I-V$ curves at scan rates varying from 5 to $500 \mathrm{mV} \mathrm{s}^{-1}$. (f) Galvanostatic charge-discharge (GCD) curves of the device at various current densities of $2-40 \mathrm{~mA} \mathrm{~cm}^{-2}$. (g) Device Nyquist plot. (h) Areal capacity of the device as a function of current density. (i) Comparison of the device's areal capacity with other values reported in previous studies. (j) Ragone plot of the energy-power density of various devices reported in the literature. (k) Successful energizing of a digital watch by the supercapacitor even under bending deformation.

charging system can be directly charged without a direct physical connection to an external power source. Therefore, a wireless power transmission system coupled with a rechargeable supercapacitor was prepared to achieve this wireless function. The input and output direct current (DC) voltages were set to 5 and $1 \mathrm{~V}$, respectively, considering that the configuration used in most portable power sources in our daily life is $5 \mathrm{~V}$. The power efficiency is highly dependent on two main factors, namely, the working distance between primary and secondary coils and the bending degree of the secondary coils (Fig. 6a). The tested working distances ranged from 0 to $15 \mathrm{~mm}$, with glass sheets used as spacers. As the working distance increased, the voltage decreased slowly, eventually dropping to $50 \%$ voltage retention when the distance was set to $7 \mathrm{~mm}$. The voltage retention followed a similar trend when the secondary coil was bent from $0^{\circ}$ to $180^{\circ}$ along its center. Even as the device was subjected to $90^{\circ}$ bending deformation, the sensor still possessed $80 \%$ retention, demonstrating its applicability under bending deformation.

The wireless power transmission was further connected to the supercapacitor to test its operational potential. The charging and discharging curves with the sensor as the working load are shown in Fig. 6b. The sensor exhibited an ultrafast charging ability (inset in Fig. 6b) when the planar distance was set to $5 \mathrm{~mm}$ because of the combined benefits of superior and efficient wireless power transmission, the high rate capability of the supercapacitor, and the ultra-low energy consumption of the sensor. Additionally, the voltage of the supercapacitor was recorded to test its self-discharging property and long-term durability. The energy storage device could retain $50 \%$ of its voltage even after $5000 \mathrm{~s}$ (more than $1 \mathrm{~h}$ ), demonstrating its long-term operating ability. The practical application of successfully driving a digital watch is shown in Fig. $6 \mathrm{c}$ and Movie S3. The wireless transmission system effectively sent the energy to the supercapacitor and 

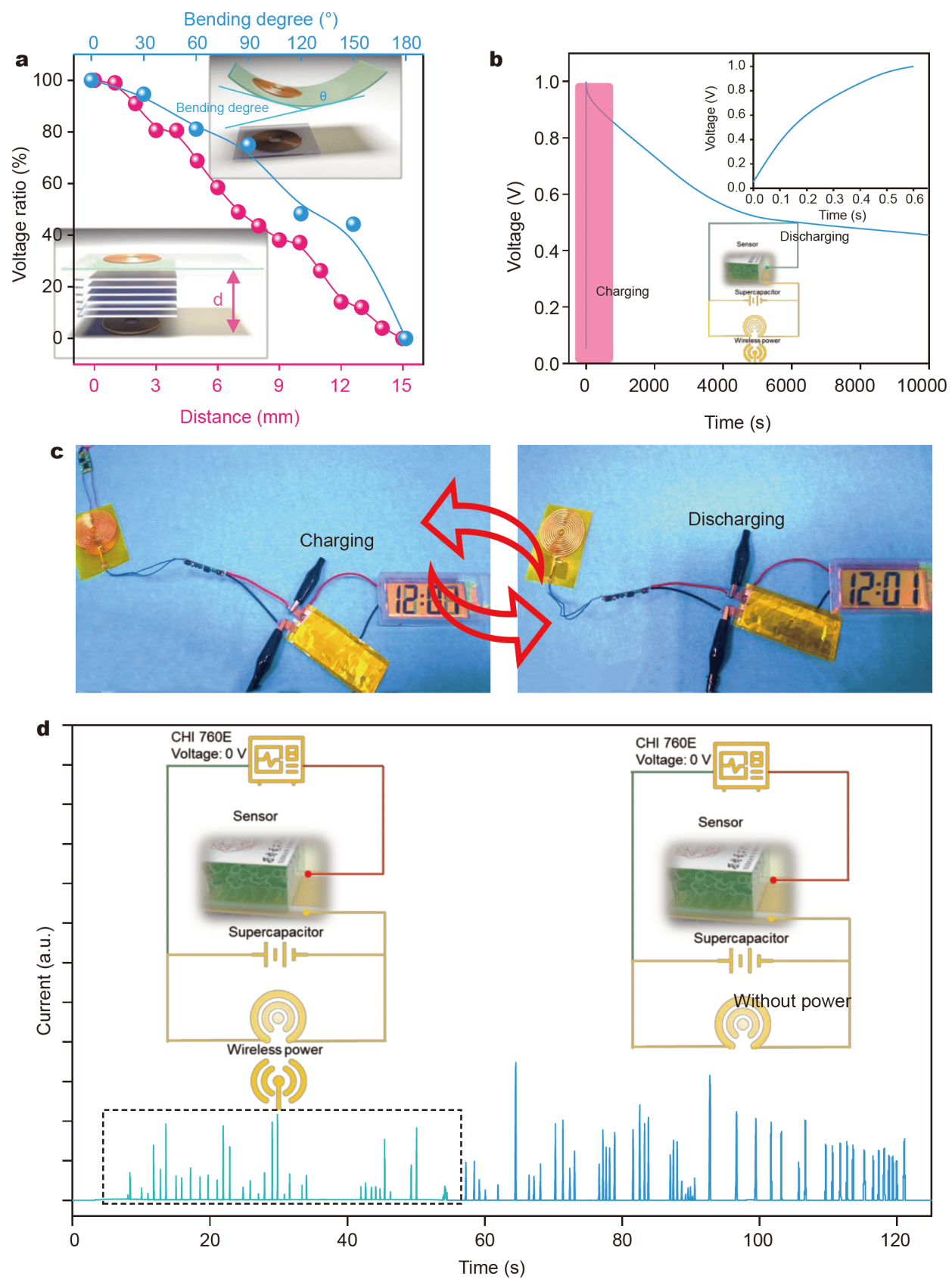

Figure 6 Wireless charging system to drive the sensor. (a) Voltage retention of the inductor coil with various bending degrees and transmission distances. (b) Charging and discharging curves of the wireless system with a working distance of $5 \mathrm{~mm}$. (c) Successful demonstration of energizing a digital watch via the wireless charging system. (d) Successful wireless powering of the sensor system for periods of interest in applications.

successfully retained the power as a reservoir for further use. In this case, the watch was continuously working even when it was removed from the charging source. Lastly, when the sensor was powered by the wireless transmission system, mechanical pressure fluctuations were successfully monitored (Fig. 6d and Movie S4).
Specifically, the sensor initially monitored the variations in finger pressure when the supercapacitor was charging (inset of Fig. 6d). After wireless charging for $45 \mathrm{~s}$, the power was withdrawn from the supercapacitor, but the sensor could function for a reasonably long time, demonstrating its potential for applications of interest. 


\section{CONCLUSIONS}

A conductive GF/PCN-coated porous elastomer with MPAs as sensing materials was successfully fabricated through magnetic-field induction coupled with a sacrificial template. The resulting hierarchically structured and porous flexible sensor not only displayed an ultrahigh sensitivity of over $10,000 \mathrm{kPa}^{-1}$ but also exhibited a low detection limit of $0.25 \mathrm{~Pa}$, a broad working range up to $500 \mathrm{kPa}$, a millisecond fast response time, and long-time durability. However, the combined high-level performance has not been reported. Additionally, the developed wireless recharging system achieved high-energy storage and transmission efficiency when it was subjected to bending deformation, allowing it to be rationally integrated with the sensor to develop various functional applications without a direct physical connection. The wirelessly powered high-performance sensor created and demonstrated in this work presented a novel strategy to construct WSNs with a breakthrough performance for future $5 \mathrm{G}$ communication and IoT applications. Furthermore, the concepts explored for sensor optimization provide a basis for investigating the incorporation of hierarchical hybrid structures and ultrasensitive flexible sensors integrated with wireless recharging systems into other flexible electronics systems. Thus, novel multifunctional applications may be developed.

Received 6 December 2020; accepted 4 February 2021; published online 22 April 2021

1 Rogers JA, Someya T, Huang Y. Materials and mechanics for stretchable electronics. Science, 2010, 327: 1603-1607

2 Wang $\mathrm{C}, \mathrm{Li} \mathrm{X}, \mathrm{Hu} \mathrm{H}$, et al. Monitoring of the central blood pressure waveform via a conformal ultrasonic device. Nat Biomed Eng, 2018, 2: 687-695

$3 \mathrm{Pu} \mathrm{X}$, Guo $\mathrm{H}$, Chen J, et al. Eye motion triggered self-powered mechnosensational communication system using triboelectric nanogenerator. Sci Adv, 2017, 3: e1700694

4 Zhu M, Sun Z, Zhang Z, et al. Haptic-feedback smart glove as a creative human-machine interface (HMI) for virtual/augmented reality applications. Sci Adv, 2020, 6: eaaz8693

5 Boutry CM, Negre M, Jorda M, et al. A hierarchically patterned, bioinspired e-skin able to detect the direction of applied pressure for robotics. Sci Robot, 2018, 3: eaau6914

6 Ran W, Wang L, Zhao S, et al. An integrated flexible all-nanowire infrared sensing system with record photosensitivity. Adv Mater, 2020, 32: 1908419

7 Zhang C, Ye WB, Zhou K, et al. Bioinspired artificial sensory nerve based on nafion memristor. Adv Funct Mater, 2019, 29: 1808783

8 Chen YL, Liu D, Wang S, et al. Self-powered smart active RFID tag integrated with wearable hybrid nanogenerator. Nano Energy, 2019, 64: 103911

9 Yue Y, Yang Z, Liu N, et al. A flexible integrated system containing a microsupercapacitor, a photodetector, and a wireless charging coil. ACS Nano, 2016, 10: 11249-11257
10 Yao S, Ren P, Song R, et al. Nanomaterial-enabled flexible and stretchable sensing systems: Processing, integration, and applications. Adv Mater, 2020, 32: 1902343

11 Lee Y, Park J, Cho S, et al. Flexible ferroelectric sensors with ultrahigh pressure sensitivity and linear response over exceptionally broad pressure range. ACS Nano, 2018, 12: 4045-4054

12 Liu K, Song W, Deng Y, et al. Electrooxidation enables highly regioselective dearomative annulation of indole and benzofuran derivatives. Nat Commun, 2020, 11: 3-11

13 Li S, Zhang Y, Wang Y, et al. Physical sensors for skin-inspired electronics. InfoMat, 2020, 2: 184-211

14 Xia K, Wang C, Jian M, et al. CVD growth of fingerprint-like patterned 3D graphene film for an ultrasensitive pressure sensor. Nano Res, 2018, 11: 1124-1134

15 Jian M, Xia K, Wang Q, et al. Flexible and highly sensitive pressure sensors based on bionic hierarchical structures. Adv Funct Mater, 2017, 27: 1606066

16 Wang K, Lou Z, Wang L, et al. Bioinspired interlocked structureinduced high deformability for two-dimensional titanium carbide (MXene)/natural microcapsule-based flexible pressure sensors. ACS Nano, 2019, 13: 9139-9147

17 Xu S, Zhang Y, Cho J, et al. Stretchable batteries with self-similar serpentine interconnects and integrated wireless recharging systems. Nat Commun, 2013, 4: 1543-1548

18 Lin Y, Chen J, Tavakoli MM, et al. Printable fabrication of a fully integrated and self-powered sensor system on plastic substrates. Adv Mater, 2019, 31: 1804285

19 Yu L, Yi Y, Yao T, et al. All VN-graphene architecture derived selfpowered wearable sensors for ultrasensitive health monitoring. Nano Res, 2019, 12: 331-338

20 Lu Y, Jiang K, Chen D, et al. Wearable sweat monitoring system with integrated micro-supercapacitors. Nano Energy, 2019, 58: 624-632

21 Gao L, Song J, Surjadi JU, et al. Graphene-bridged multifunctional flexible fiber supercapacitor with high energy density. ACS Appl Mater Interfaces, 2018, 10: 28597-28607

22 Gao L, Wang Y, Hu X, et al. Cellular carbon-film-based flexible sensor and waterproof supercapacitors. ACS Appl Mater Interfaces, 2019, 11: 26288-26297

23 Song Y, Chen H, Chen X, et al. All-in-one piezoresistive-sensing patch integrated with micro-supercapacitor. Nano Energy, 2018, 53: $189-197$

24 Song Y, Chen H, Su Z, et al. Highly compressible integrated supercapacitor-piezoresistance-sensor system with CNT-PDMS sponge for health monitoring. Small, 2017, 13: 1702091

25 Zhou Y, Chen C, Zhu S, et al. A printed, recyclable, ultra-strong, and ultra-tough graphite structural material. Mater Today, 2019, 30: $17-25$

$26 \mathrm{Xu} \mathrm{H}$, Gao L, Wang Y, et al. Flexible waterproof piezoresistive pressure sensors with wide linear working range based on conductive fabrics. Nano-Micro Lett, 2020, 12: 159

27 Ge J, Wang X, Drack M, et al. A bimodal soft electronic skin for tactile and touchless interaction in real time. Nat Commun, 2019, 10: 4405

28 Lee B, Oh JY, Cho H, et al. Ultraflexible and transparent electroluminescent skin for real-time and super-resolution imaging of pressure distribution. Nat Commun, 2020, 11: 663

29 Huang J, Peng S, Gu J, et al. Self-powered integrated system of a strain sensor and flexible all-solid-state supercapacitor by using a high performance ionic organohydrogel. Mater Horiz, 2020, 7: 
2085-2096

30 Wu C, Zhang T, Zhang J, et al. A new approach for an ultrasensitive tactile sensor covering an ultrawide pressure range based on the hierarchical pressure-peak effect. Nanoscale Horiz, 2020, 5: 541-552

31 Chen M, Hu X, Li K, et al. Self-assembly of dendritic-lamellar MXene/carbon nanotube conductive films for wearable tactile sensors and artificial skin. Carbon, 2020, 164: 111-120

32 Zhang L, Liu X, Zhong M, et al. Micro-nano hybrid-structured conductive film with ultrawide range pressure-sensitivity and bioelectrical acquirability for ubiquitous wearable applications. Appl Mater Today, 2020, 20: 100651

33 Tang X, Wu C, Gan L, et al. Multilevel microstructured flexible pressure sensors with ultrahigh sensitivity and ultrawide pressure range for versatile electronic skins. Small, 2019, 15: 1804559

34 Xiao Z, Zhou W, Zhang N, et al. All-carbon pressure sensors with high performance and excellent chemical resistance. Small, 2019, 15: 1804779

35 Li H, Wu K, Xu Z, et al. Ultrahigh-sensitivity piezoresistive pressure sensors for detection of tiny pressure. ACS Appl Mater Interfaces, 2018, 10: 20826-20834

36 Yuan L, Wang Z, Li H, et al. Synergistic resistance modulation toward ultrahighly sensitive piezoresistive pressure sensors. Adv Mater Technol, 2020, 5: 1901084

37 Feng B, Zou G, Wang W, et al. A programmable, gradient-composition strategy producing synergistic and ultrahigh sensitivity amplification for flexible pressure sensing. Nano Energy, 2020, 74: 104847

38 Deng C, Tang W, Liu L, et al. Self-powered insole plantar pressure mapping system. Adv Funct Mater, 2018, 28: 1801606

39 Lu AK, Li HY, Yu Y. Holey graphene synthesized by electrochemical exfoliation for high-performance flexible microsupercapacitors. J Mater Chem A, 2019, 7: 7852-7858

40 Qi H, Yick S, Francis O, et al. Nanohybrid TiN/vertical graphene for high-performance supercapacitor applications. Energy Storage Mater, 2020, 26: 138-146

41 Pham TV, Kim JG, Jung JY, et al. High areal capacitance of Ndoped graphene synthesized by arc discharge. Adv Funct Mater, 2019, 29: 1905511

42 Zhao J, Zhang Y, Huang Y, et al. Duplex printing of all-in-one integrated electronic devices for temperature monitoring. J Mater Chem A, 2019, 7: 972-978

43 Zheng S, Li Z, Wu ZS, et al. High packing density unidirectional arrays of vertically aligned graphene with enhanced areal capacitance for high-power micro-supercapacitors. ACS Nano, 2017, 11: 4009-4016

44 El-Kady MF, Kaner RB. Scalable fabrication of high-power graphene micro-supercapacitors for flexible and on-chip energy storage. Nat Commun, 2013, 4: 1475-1479

45 Guo H, Liu L, Dou Q, et al. Punching holes on paper-like electrodes: An effective strategy to enhance rate performance of supercapacitors. Energy Storage Mater, 2019, 19: 338-345

46 Li J, Sollami Delekta S, Zhang P, et al. Scalable fabrication and integration of graphene microsupercapacitors through full inkjet printing. ACS Nano, 2017, 11: 8249-8256

47 Ling $\mathrm{Y}$, Zhuang $\mathrm{X}, \mathrm{Xu} \mathrm{Z}$, et al. Mechanically assembled, threedimensional hierarchical structures of cellular graphene with programmed geometries and outstanding electromechanical properties. ACS Nano, 2018, 12: 12456-12463

48 Huang X, Wu P. A facile, high-yield, and freeze-and-thaw-assisted approach to fabricate MXene with plentiful wrinkles and its application in on-chip micro-supercapacitors. Adv Funct Mater, 2020, 30: 1910048

49 Shao Y, Li J, Li Y, et al. Flexible quasi-solid-state planar microsupercapacitor based on cellular graphene films. Mater Horiz, 2017, 4: 1145-1150

50 Yu J, Wang M, Xu P, et al. Ultrahigh-rate wire-shaped supercapacitor based on graphene fiber. Carbon, 2017, 119: 332-338

51 Zhang L, DeArmond D, Alvarez NT, et al. Flexible micro-supercapacitor based on graphene with 3D structure. Small, 2017, 13: 1603114

52 Ramadoss A, Yoon KY, Kwak MJ, et al. Fully flexible, lightweight, high performance all-solid-state supercapacitor based on 3dimensional-graphene/graphite-paper. J Power Sources, 2017, 337: 159-165

53 Manjakkal L, Núñez CG, Dang W, et al. Flexible self-charging supercapacitor based on graphene-Ag-3D graphene foam electrodes. Nano Energy, 2018, 51: 604-612

Acknowledgements This work was supported by the National Natural Science Foundation of China (61904141), the Funding of the Natural Science Foundation of Shaanxi Province (2020JQ-295), China Postdoctoral Science Foundation (2020M673340), the Key Research and Development Program of Shaanxi (2020GY-252), and the National Key Laboratory of Science and Technology on Vacuum Technology and Physics (HTKJ2019KL510007).

Author contributions Gao L designed and fabricated the samples, conducted the experiments, analyzed the data, and wrote the manuscript. Han Y analyzed the data, conducted the experiments, and drafted a part of the manuscript. Surjadi JU wrote a part of the manuscript and revised it. Zhou $\mathrm{W}$ wrote a part of the manuscript, and Cao K performed the SEM experiments. Gao L, Wang W, and Espinosa HD led this project. All authors reviewed and approved the final version of the paper.

Conflict of interest These authors declare no conflict of interest.

Supplementary information Supporting data are available in the online version of the paper.

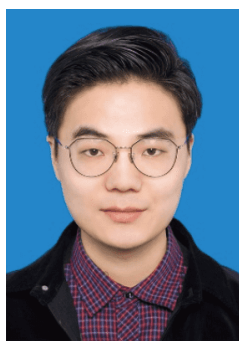

Libo Gao received his $\mathrm{PhD}$ degree from the City University of Hong Kong (CityU) in 2018. He continued working as a Research Assistant at CityU before he joined Xidian University. Now he is an associate professor at Xidian University. His research mainly focuses on intelligent micro/ nano electronic devices and micro systems. 


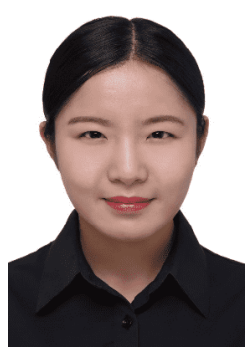

Ying Han received her Bachelor's degree from Tianjin University in 2017. Now she is a $\mathrm{PhD}$ candidate at the Department of Mechanical Engineering, City University of Hong Kong. Her research focuses on nanomechanics of low-dimensional materials and hierarchical structures such as hierarchically micro-structured sensor devices.

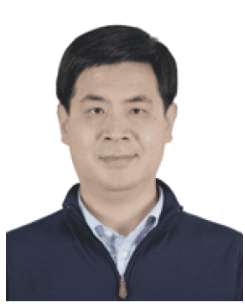

Weidong Wang received his $\mathrm{BSc}, \mathrm{MSc}$ and $\mathrm{PhD}$ degrees in mechanical engineering from Xidian University, where he is currently a professor and the director of the Research Center of MicroNano Systems. His research interests include MEMS/NEMS, micro-nano manufacturing, and micro-nano mechanics.

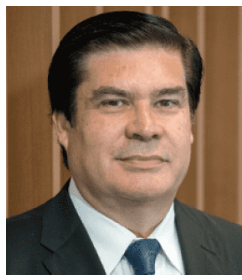

Horacio D. Espinosa received his $\mathrm{PhD}$ degree from Brown University. Now he is a professor at the Northwestern University. His research mainly focuses on the mechanical behavior of natural and synthetic nanomaterials and micro/ nano devices for materials research and personalized medicine.

\section{基于磁诱导微柱阵列可无线充电的超灵敏柔性传} 感器

高立波 ${ }^{1,2,3 \dagger^{*}}$, 韩英 ${ }^{4 \dagger}$, James Utama Surjadi ${ }^{4}$, 曹可 ${ }^{1,2}$, 周文钊 ${ }^{3}$, 徐洪成 ${ }^{1,2}$, 胡新康 ${ }^{1,2}$, 王明智 ${ }^{1,2}$, 㚞康旗 ${ }^{1,2}$, 王月皎 ${ }^{3,4}$, 王卫东 ${ }^{1,2,5^{*}}$, Horacio D. Espinosa ${ }^{5,6^{*}}$

摘要 为实现在宽感知范围内对微小压力变化的精确测量, 提高柔 性压力传感器的灵敏度和工作范围显得尤为迫切. 然而, 由于柔性 传感器所使用的软基质材料的固有局限, 实现超过 $100 \mathrm{kPa}$ 工作范 围同时维持 $1000 \mathrm{kPa}^{-1}$ 以上的灵敏度仍然是一项挑战. 本文报道了 一种磁诱导的多孔弹性体(PDMS), 分别以微柱阵列(MPA)作为传 感材料和具有编织结构的导电镍作为电极. 由于 MPA的独特结构 和电极的织构形态, 所开发的传感器具有 $10,268 \mathrm{kPa}^{-1}(0.6-170 \mathrm{kPa}$ 范围内）的超高灵敏度, 高达 $500 \mathrm{kPa}$ 的工作范围, 并具有长期耐用 性. 并实现了最小 $0.25 \mathrm{~Pa}$ 的检测压力和 $3 \mathrm{~ms}$ 的响应时间. 此外, 将柔 性传感器、柔性超级电容器和感应线圈进行一体化集成, 实现了 对传感器的无线能量传输, 并在低压至高压范围内对传感实际应 用能力进行了测试. 本项研究工作将具有微结构的高性能传感器 与无线充电系统结合在一起, 为开发下一代柔性电子产品提供了 一种新颖的方法. 